\title{
Globally Convergent Nonlinear 3D Inverse Model for Smart Materials with Hessian-based Optimization
}

\author{
Hafez Tari $^{1}$, Marcelo J. Dapino ${ }^{2}$ \\ I/UCRC on Smart Vehicle Concepts, Department of Mechanical and Aerospace Engineering, The Ohio State \\ University, Columbus, $\mathrm{OH}$ 43210, USA
}

\begin{abstract}
A globally convergent and fully coupled 3D inverse model for smart materials is presented. In practice, stress and field (electric, magnetic, or temperature) are applied to smart materials whereas strain and flux density (electric, magnetic, or temperature) are measured. We refer to constitutive models that follow this scheme as direct models. In certain design and control situations, however, inverse models are necessary in which the field and stress are found from specified flux density and strain. This inversion typically involves an iterative procedure, which may be prone to convergence issues. An inverse model approach is proposed for arbitrary smart materials. The inversion requirement is a continuous and second order differentiable direct model for any chosen smart material. The approach is globally convergent, which makes it ideal for use in finite element frameworks. The premise of the proposed iterative system model is to constitute a recursive correction formula based on second order approximations of a novel scalar error function which offers a faster convergence rate. A continuation approach is then used to achieve global convergence for arbitrary input parameters. Magnetostrictive Galfenol is chosen to illustrate the effectiveness of the inverse model, and compact analytical derivations of the Jacobian and Hessian matrices are presented. The convergence rate of the proposed approach is superior to that of an existing inverse model. Finally, the inverse model's robustness is demonstrated through integration of the model into a finite-element framework to simulate a magnetostrictive composite plate actuator in full 3D.
\end{abstract}

Keywords:

Inverse Model, Active Composite Plate, Iron-Gallium Alloy, Galfenol, Constitutive Model, Magnetostriction.

\footnotetext{
${ }^{1}$ E-mail: tari.1@osu.edu

${ }^{2}$ Address all correspondence to this author. E-mail: dapino.1@osu.edu
} 


\section{Introduction}

Smart materials convert energy from one type to another, resulting in controllable changes in material properties along with sensing, actuation, and other forms of coupled response. For instance, piezoelectric and electrostrictive materials convert electrical energy to mechanical energy and vice versa; magnetostrictive materials exhibit coupling of magnetic and mechanical effects; shape-memory alloys and polymers respond to thermal activation by changing their crystal structure and macroscopic dimensions; and $\mathrm{pH}$ sensitive polymers swell in response to changes in external pH. See Smith [1] and Leo [2] for more information on smart materials.

Smart materials typically exhibit nonlinear and hysteretic behavior, which varies substantially between the materials. For instance, when piezoelectric materials like PZT, PVDF, and $\mathrm{BaTiO}_{3}$ are poled, they exhibit approximately linear behavior for low to moderate inputs [3]. These materials can be efficiently characterized using linear models within a specified range of operation. In contrast, shape memory materials, electrostrictives, and magnetostrictives exhibit saturation nonlinearity and hysteresis, thus accurate description of their behavior requires requiring complex constitutive models. Nonlinear smart material models have been formulated considering phenomenological, microscopic, and macroscopic viewpoints. Phenomenological models fit a curve or surface to measurement data, which provides efficiency but ignores the underlying physics. In contrast, microscopic models provide a more accurate description of the physical phenomena by considering all possible energies. This, however, tends to entail high complexity and computational cost. On the other hand, macroscopic models use an intermediate approach by relating the bulk response of a material to simplified descriptions of its microscopic behavior. This approach strikes a balance between computational speed, accuracy, and generality.

Many different models have been developed for smart materials. For instance, Stebner and Brinson [4] presented a 3D constitutive model that describes evolution of elastic and transformation strains during thermo-mechanical shape memory alloy loading events assuming a symmetric, isotropic material response. See Cisse et al. [5] for a very recent review of constitutive models and modeling techniques for shape memory alloys. Keip et al. [6] presented a two-scale computational homogenization framework for the simulation of electro-active solids at finite strains. Linder and Zhang [7] presented 3D finite elements with embedded strong discontinuities to model failure in electromechanical coupled materials. Zheng and Zhang [8] studied adaptive finite-element methods for time-harmonic eddy current problems in the case of 3D isotropic and linear magnetic materials. Greif et al. [9] developed a mixed finite-element method for the 
numerical discretization of 2D and 3D stationary incompressible magnetohydrodynamics problems. Caloz et al. [10] studied the influence of geometry on the skin effect in electromagnetism. Dong et al. [11] analyzed convergence and stability of three finite-element iterative methods for 2D and 3D stationary incompressible magnetohydrodynamics.

Terbium-dysprosium-iron (Terfenol-D) and iron-gallium (Galfenol) are the recent magnetostrictive materials. Terfenol-D produces relatively large magnetostriction $(\approx 1600 \mathrm{ppm})$ at a moderate magnetic field $(\approx 200 \mathrm{kA} / \mathrm{m})$, making it well suited for actuator designs; see for example Chakrabarti and Dapino [12]. Because Terfenol-D is brittle, it always requires stress biasing to avoid tension. On the other hand, Galfenol exhibits moderate magnetostriction $(\approx$ $350 \mathrm{ppm})$ at low magnetic fields $(\approx 8 \mathrm{kA} / \mathrm{m})$, possesses high tensile strength $(\approx 500 \mathrm{MPa})$, and experiences a limited variation in magnetomechanical properties for temperatures between -20 and $80{ }^{\circ} \mathrm{C}$ (see Ref. [13]). When composed of less than 20 at.\% gallium, Galfenol retains the machinability and ductility of iron. As such, it can be produced in sheets or wires, as well as welded, threaded or extruded into unprecedented 3D structures that are both active and load-bearing. Several techniques were proposed in micromagnetics to solve the Landau-Lifshitz equation [14] that describes the evolution of magnetization in a ferromagnetic material. For example, Gauss-Seidel projection method was proposed by Wang et al. [15] and a boundarycorrected algorithm for general geometries was developed by García-Cervera et al. [16]. On the other hand, the Preisach model [17], Globus model [18], Jiles-Atherton model [19], and StonerWohlfarth model [20] are some of the classical models for magnetostrictives. Liorzou et al. [21] compared the pros and cons of these models in detail. For Terfenol-D, Carman and Mitrovic [22] formulated a model by expanding the Gibbs free energy in a truncated Taylor series, the coefficients of which were determined experimentally. Zheng and Sun [23] included higher order terms in the expansion to improve the applicability of the model for larger magnetic field inputs. Armstrong [24] proposed an incremental hysteretic magnetoelastic constitutive theory of pseudo-cubic magnetostrictive alloys that can be applied to Terfenol-D and, with some modifications, to Galfenol. The Armstrong model is computationally intensive, as it searches for global energy minima. Evans and Dapino [25], and later Chakrabarti [26], greatly enhanced the computational speed by formulating the energy functions around local minima along Galfenol's six easy crystallographic directions.

In practice, stress and field (electric, magnetic, or thermal) are the independent variables to characterize smart materials, and strain and flux density (electric, magnetic, or thermal) are the 
dependent variables, which would be measured. We refer to the constitutive models that follow this scheme as direct models in a mathematical sense. In certain design and control situations, inverse models are necessary, wherein stresses and fields must be determined from specified strains and flux densities. This inversion typically involves an iterative procedure, and requires sophisticated criteria to find physical solutions, especially, when multiple mathematical solutions exist. Chakrabarti and Dapino [27] proposed an inverse model that describes the full nonlinear coupling in 3D magnetic transducers. However, this model is susceptible to convergence issues, which are drastically alleviated by further developments presented by Deng and Dapino [28]. Nonetheless, both of these models are built on the direct model given in Ref. [26], which is prone to singularities. These can burden computation, especially when the model is integrated into finite-element solvers. Tari et al. [29] addressed this shortcoming through a reformulation of this direct model with an exact solution procedure. In addition, Tari et al. [30] recently presented an efficient inverse model for a partially coupled magnetomechanical system, in which stresses must be found for given field values. The model was successfully applied to a composite magnetostrictive plate operating in actuation mode.

The goal of this paper is to generalize the aforementioned pieces of work and present a fast and globally convergent inverse model for fully coupled and arbitrary smart materials with any given direct models. Our strategy to have such an inverse model rests on "continuation". The idea of continuation on the solution of a highly nonlinear problem is to break the problem (say, the target problem) into a series of more manageable subproblems through a subdivision of the underlying parameters, and solve them sequentially. That is, the parameters are subdivided into a series of ordered subsets, each of which would define a subproblem. The first subset specifies the start subproblem, and the last subset determines the target problem. The subdivision is generally done such that the start subproblem is relatively much less intense to solve. The solution obtained for the start subproblem is marked as the start solution, and is used for the solution of the subsequent subproblem using an iterative approach. This is repeated until the solution to the target problem is obtained. The idea of continuation has been employed in a variety of disciplines. For instance, Tari et al. [31, 32] applied continuation to kinematic design and analysis of rigid mechanisms. Accordingly, a significant speed in the design process was achieved through avoiding the computation for the majority of mathematically valid solutions, which are in fact nonphysical solutions. Additionally, Banerjee et al. [33] presented a continuation based approach for large-scale inverse identification of linear elastic material properties in the context of steady- 
state elastodynamics. They demonstrated that their approach can significantly accelerate the solution convergence. Ligurský and Renard [34] recently presented a continuation algorithm for the solution of discretized evolution problems with application to plane quasi-static contact problems with friction. A set of model examples was used to successfully demonstrate their algorithm's performance.

The aforementioned existing inverse models constitute recursive correction formulae based on first order approximations of some specified error functions. However, the aim of this paper is to achieve a faster convergence rate by taking second order approximations into account. To do this, we formulate the problem in an optimization framework through the definition of a novel scalar error function, which allows to effectively incorporate Hessian (matrix of the second order derivatives) of the direct model in the formulation. Thus, the inversion requirement is a continuous and second order differentiable direct model for any chosen smart material. The resulting approach is globally convergent, which makes it ideal for use in finite-element frameworks. The proposed methodology generalizes that of Tari and Dapino [35], specialized for magnetostrictives, to arbitrary smart materials. To illustrate the inverse model, the magnetostrictive Galfenol is chosen and compact analytical derivations of the Jacobian and Hessian matrices corresponding to the direct model given by Tari et al. [29] are presented. The proposed model's effectiveness is compared successfully to that of Deng and Dapino [28] for the chosen material with 1D input data. Additionally, the inverse model is integrated into a finite-element framework to simulate an aluminum-Galfenol composite plate actuator. Tari et al. [30] recently presented a reduced $2 \mathrm{D}$ model for this composite plate with the assumption that the magnetic field distribution throughout the Galfenol volume is approximately uniform for the small deformations, thus they assumed that the magnetic field is known. However, we lift this assumption in this work, and simulate this composite plate actuator in full 3D.

The rest of the paper is organized as follows. A globally convergent inverse model for arbitrary smart materials is outlined next. A brief review of a recent direct model for magnetostrictive Galfenol is given in Section 3. The first and second order derivative terms of this direct model, required by the proposed inverse model, are derived analytically in Section 4 . The performance of the inverse model is benchmarked for special and general input data; model's insensitivity to special input data is compared with that of an existing inverse model in Section 5 with 1D input data, and its robustness for general input data is demonstrated in Section 6 with a fully coupled, 3D simulation of a magnetostrictive composite plate actuator. Finally, conclusions 
are given.

\section{Smart Material Inverse Model}

Let $\mathbf{T}$ and $\mathcal{F}$ denote, respectively, stress and field (electric, magnetic, or thermal), and $\mathbf{S}(\mathcal{F}, \mathbf{T})$ and $\mathbf{B}(\mathcal{F}, \mathbf{T})$ denote, respectively, strain and flux density (electric, magnetic, or thermal), which constitute a given direct model for any arbitrary smart material. Also, let $\mathbf{B}^{*}$ and $\mathbf{S}^{*}$ denote any discrete flux and strain tensors specified from measurements or finite-element simulations. In addition, for the sake of convenience, let all of the tensors be written in an equivalent vector form. For example, the symmetric stress tensor is written as $\mathbf{T}=\left[T_{1} ; T_{2} ; T_{3} ; T_{4} ; T_{5} ; T_{6}\right]$ with the convention that $T_{1}=T_{11}, T_{2}=T_{22}, T_{3}=T_{33}, T_{4}=T_{12}, T_{5}=T_{23}$, and $T_{6}=T_{13}$.

The goal is to find the unknown field $\left(\mathcal{F}^{*}\right)$ and stress $\left(\mathbf{T}^{*}\right)$ that give rise to $\mathbf{B}^{*}$ and $\mathbf{S}^{*}$. In other words, the goal is to find $\mathcal{F}$ and $\mathbf{T}$ that satisfy the equations

$$
\left[\begin{array}{l}
\mathbf{B}(\mathcal{F}, \mathbf{T})-\mathbf{B}^{*} \\
\mathbf{S}(\mathcal{F}, \mathbf{T})-\mathbf{S}^{*}
\end{array}\right]=0
$$

It is well known that if a small perturbation is made to the input parameters of a system of equations, then the solutions to the perturbed system will perturb only slightly. This is the premise of the continuation technique, thus we use it to ensure global convergence in solving the foregoing system of equations (see, e.g., Refs. [31] and [32] for more on continuation). Let us rewrite the foregoing vector of error functions as a linear homotopy

$$
\left[\begin{array}{c}
\mathbf{B}(\mathcal{F}, \mathbf{T})-\mathbf{B}^{\tau} \\
\mathbf{S}(\mathcal{F}, \mathbf{T})-\mathbf{S}^{\tau}
\end{array}\right] \triangleq\left[\begin{array}{c}
\mathbf{B}(\mathcal{F}, \mathbf{T})-\left((1-\tau) \mathbf{B}\left(\mathcal{F}^{0}, \mathbf{T}^{0}\right)+\tau \mathbf{B}^{*}\right) \\
\mathbf{S}(\mathcal{F}, \mathbf{T})-\left((1-\tau) \mathbf{S}\left(\mathcal{F}^{0}, \mathbf{T}^{0}\right)+\tau \mathbf{S}^{*}\right)
\end{array}\right]=0
$$

where $\mathcal{F}^{0}$ and $\mathbf{T}^{0}$ are known start solutions, and $\tau \in[0,1]$ is the continuation parameter. Continuation initiates at the start solutions $\mathcal{F}^{0}$ and $\mathbf{T}^{0}$ at $\tau=0$, and traces the solution curves of $\mathcal{F}(\tau)$ and $\mathbf{T}(\tau)$ as $\tau$ is incremented, until $\tau=1$ at which point the desired solutions $\mathcal{F}^{*}$ and $\mathbf{T}^{*}$ are obtained.

To solve each subproblem effectively, we solve the minimization problem

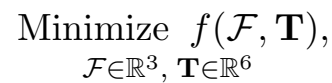

where $\mathbb{R}$ is the set of the real numbers, and $f$ is the scalar, continuous objective error function defined as 


$$
f(\mathcal{F}, \mathbf{T}) \triangleq \frac{1}{2} w_{b}^{2}\left[\mathbf{B}(\mathcal{F}, \mathbf{T})-\mathbf{B}^{\tau}\right]^{\mathrm{T}}\left[\mathbf{B}(\mathcal{F}, \mathbf{T})-\mathbf{B}^{\tau}\right]+\frac{1}{2} w_{s}^{2}\left[\mathbf{S}(\mathcal{F}, \mathbf{T})-\mathbf{S}^{\tau}\right]^{\mathrm{T}}\left[\mathbf{S}(\mathcal{F}, \mathbf{T})-\mathbf{S}^{\tau}\right]
$$

where []$^{T}$ is the vector or matrix transpose operator, and $w_{b}$ and $w_{s}$ are weighting factors chosen to effectively combine the error contributions of magnetic flux density and strain, respectively. With [; ] as column vector operator, and expanding $f$ in a second order Taylor's series as

$f(\mathcal{F}+\Delta \mathcal{F}, \mathbf{T}+\Delta \mathbf{T}) \approx$

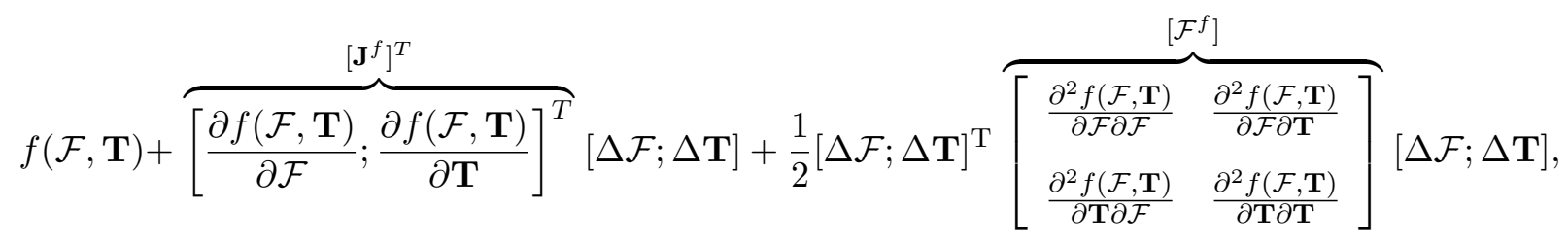

and minimizing it for the incremental field and stress vectors $\Delta \mathcal{F}$ and $\Delta \mathbf{T}$, may give a recursive correction formula based on the damped Newton method as

$$
[\mathcal{F}(i+1) ; \mathbf{T}(i+1)]=[\mathcal{F}(i) ; \mathbf{T}(i)]-\alpha_{i}\left[\mathcal{F}^{f}(i)\right]^{-1}\left[\mathbf{J}^{f}(i)\right]
$$

where $i$ is the iteration index, $\alpha_{i}$ is the step size, and $\mathbf{J}^{f}$ and $\mathcal{F}^{f}$ are called the Jacobian (gradient vector for 1D inputs) and Hessian matrices, respectively. When the derivative terms are known, the algorithm initiates at given start solutions $\mathcal{F}(0)$ and $\mathbf{T}(0)$, which get corrected at successive iterations, until the algorithm is terminated when the residual error is below a predetermined threshold. At this point, the desired $\mathcal{F}^{\tau}$ and $\mathbf{T}^{\tau}$ are obtained for each subproblem. Note that the notion of time is implicit to the increments $\Delta \mathcal{F}$ and $\Delta \mathbf{T}$. Therefore, in a dynamics case, these increments need to be small enough to capture the dynamics of the system.

For convenience, the derivatives in eq. (5) or (6) are derived in indicial where it is assumed that the subscripts $p, q \in\{1, \ldots, 3\}$ and $i, j \in\{1, \ldots, 6\}$. For brevity, $f(\mathcal{F}, \mathbf{T}), \mathbf{B}(\mathcal{F}, \mathbf{T})$ and $\mathbf{S}(\mathcal{F}, \mathbf{T})$ are abbreviated, respectively, as $f, \mathbf{B}$, and $\mathbf{S}$. Accordingly, the first order derivatives are

$$
\begin{aligned}
\frac{\partial f}{\partial \mathcal{F}_{p}} & =w_{h}\left(w_{b}^{2}\left[\mathbf{B}-\mathbf{B}^{\tau}\right]^{\mathrm{T}} \frac{\partial \mathbf{B}}{\partial \mathcal{F}_{p}}+w_{s}^{2}\left[\mathbf{S}-\mathbf{S}^{\tau}\right]^{\mathrm{T}} \frac{\partial \mathbf{S}}{\partial \mathcal{F}_{p}}\right) \\
\frac{\partial f}{\partial T_{i}} & =w_{t}\left(w_{b}^{2}\left[\mathbf{B}-\mathbf{B}^{\tau}\right]^{\mathrm{T}} \frac{\partial \mathbf{B}}{\partial T_{i}}+w_{s}^{2}\left[\mathbf{S}-\mathbf{S}^{\tau}\right]^{\mathrm{T}} \frac{\partial \mathbf{S}}{\partial T_{i}}\right)
\end{aligned}
$$


and the second order derivatives are

$$
\begin{aligned}
\frac{\partial^{2} f}{\partial \mathcal{F}_{p} \partial \mathcal{F}_{q}} & =w_{h}^{2}\left(w_{b}^{2}\left[\mathbf{B}-\mathbf{B}^{\tau}\right]^{\mathrm{T}} \frac{\partial^{2} \mathbf{B}}{\partial \mathcal{F}_{p} \partial \mathcal{F}_{q}}+w_{b}^{2} \frac{\partial \mathbf{B}^{\mathrm{T}}}{\partial \mathcal{F}_{p}} \frac{\partial \mathbf{B}}{\partial \mathcal{F}_{q}}+w_{s}^{2}\left[\mathbf{S}-\mathbf{S}^{\tau}\right]^{\mathrm{T}} \frac{\partial^{2} \mathbf{S}}{\partial \mathcal{F}_{p} \partial \mathcal{F}_{q}}+w_{s}^{2} \frac{\partial \mathbf{S}^{\mathrm{T}}}{\partial \mathcal{F}_{p}} \frac{\partial \mathbf{S}}{\partial \mathcal{F}_{q}}\right), \\
\frac{\partial^{2} f}{\partial T_{i} \partial T_{j}} & =w_{t}^{2}\left(w_{b}^{2}\left[\mathbf{B}-\mathbf{B}^{\tau}\right]^{\mathrm{T}} \frac{\partial^{2} \mathbf{B}}{\partial T_{i} \partial T_{j}}+w_{b}^{2} \frac{\partial \mathbf{B}^{\mathrm{T}}}{\partial T_{i}} \frac{\partial \mathbf{B}}{\partial T_{j}}+w_{s}^{2}\left[\mathbf{S}-\mathbf{S}^{\tau}\right]^{\mathrm{T}} \frac{\partial^{2} \mathbf{S}}{\partial T_{i} \partial T_{j}}+w_{s}^{2} \frac{\partial \mathbf{S}^{\mathrm{T}}}{\partial T_{i}} \frac{\partial \mathbf{S}}{\partial T_{j}}\right), \\
\frac{\partial^{2} f}{\partial \mathcal{F}_{p} \partial T_{i}} & =w_{h} w_{t}\left(w_{b}^{2}\left[\mathbf{B}-\mathbf{B}^{\tau}\right]^{\mathrm{T}} \frac{\partial^{2} \mathbf{B}}{\partial \mathcal{F}_{p} \partial T_{i}}+w_{b}^{2} \frac{\partial \mathbf{B}^{\mathrm{T}}}{\partial \mathcal{F}_{p}} \frac{\partial \mathbf{B}}{\partial T_{i}}+w_{s}^{2}\left[\mathbf{S}-\mathbf{S}^{\tau}\right]^{\mathrm{T}} \frac{\partial^{2} \mathbf{S}}{\partial \mathcal{F}_{p} \partial T_{i}}+w_{s}^{2} \frac{\partial \mathbf{S}^{\mathrm{T}}}{\partial \mathcal{F}_{p}} \frac{\partial \mathbf{S}}{\partial T_{i}}\right),
\end{aligned}
$$

where $w_{h}$ and $w_{t}$ are scaling factors for field and stress vectors. Note that the derivatives of the direct flux density and stress models present in the foregoing equations must be known. These terms are material specific, and, in the following section, we take Galfenol as a case study, and after a brief review of a direct model for Galfenol, the corresponding derivative terms are presented analytically.

\section{Review of Calculation of 3D Magnetostriction and Magnetic Flux Density for Galfenol}

Tari et al. [29] recently proposed an exact solution procedure for a reformulation of the discrete energy-averaged model, proposed by Evans and Dapino [25], that computes the macroscopic 3D magnetic flux density $\mathbf{B}$ and strain $\mathbf{S}$ by minimizing the Gibbs free energy that is defined locally about each easy crystallographic direction. Magnetocrystalline (anisotropy), magnetoelastic (magnetomechanical coupling), and magnetic field (Zeeman) energies constitute the Gibbs free energy in the vicinity of the $k^{\text {th }}$ easy direction written as

$$
\stackrel{k}{G}=\frac{1}{2} \stackrel{k}{\mathbf{m}} \cdot \mathbf{K} \stackrel{k}{\mathbf{m}}-\left(K \stackrel{k}{\mathbf{c}}+\mu_{0} M_{s} \mathbf{H}\right) \cdot \stackrel{k}{\mathbf{m}}+\stackrel{k}{K_{0}}, \quad k \in \pm\left\{1, \ldots, \frac{r}{2}\right\}
$$

where "." is the vector dot product operator; $K$ and $K_{0}$ are anisotropy energy constants; $\mathbf{m}=\left[m_{1} ; m_{2} ; m_{3}\right]$ is the magnetization direction having unit magnitude; $r$ is the number of easy crystallographic directions (c: the $\langle 100\rangle$ family of six directions for Galfenol); $\mu_{0}$ and $M_{s}$ are, respectively, the vacuum permeability and saturation magnetization; and the magnetic stiffness matrix is given by 


$$
\mathbf{K}=-3\left[\begin{array}{ccc}
\lambda_{100} T_{1} & \lambda_{111} T_{4} & \lambda_{111} T_{6} \\
\lambda_{111} T_{4} & \lambda_{100} T_{2} & \lambda_{111} T_{5} \\
\lambda_{111} T_{6} & \lambda_{111} T_{5} & \lambda_{100} T_{3}
\end{array}\right]
$$

where $\lambda_{100}$ and $\lambda_{111}$ are magnetostriction constants.

The macroscopic 3D magnetic flux density and strain vectors are defined as weighted sums of the response due to the $r$ minimum energy directions as

$$
\begin{aligned}
& \mathbf{B}=\mu_{0}(\mathbf{H}+\overline{\mathbf{M}})=\mu_{0}\left(\mathbf{H}+M_{s} \sum_{k= \pm 1}^{ \pm r / 2} \frac{k}{\xi_{h y s}} \underset{\mathbf{m}}{k}\right), \\
& \mathbf{S}=\mathbf{s} \mathbf{T}+\overline{\boldsymbol{\lambda}}=\mathbf{s} \mathbf{T}+\sum_{k= \pm 1}^{ \pm r / 2} \frac{k}{\bar{\xi}_{h y s}} \boldsymbol{\lambda},
\end{aligned}
$$

where $\bar{\xi}_{\text {hys }}$ and $\stackrel{k}{\lambda}$ denote, respectively, the averaged hysteretic volume fraction and the magnetostriction tensor written in vector notation for the $k^{\text {th }}$ domain; s stands for the $6 \times 6$ mechanical compliance matrix. Letting $\Omega$ be a smoothing factor, the former is calculated as a Boltzmanntype, energy-weighted average as

$$
\bar{\xi}_{h y s}=\frac{\exp \left(\begin{array}{c}
k \\
-\frac{G}{\Omega}
\end{array}\right)}{\sum_{n= \pm 1}^{ \pm r / 2} \exp \left(-\frac{G}{\Omega}\right)},
$$

and the components of the magnetostriction tensor $\stackrel{k}{\lambda}$ are

$$
\begin{aligned}
& \stackrel{k}{\lambda}_{u u}=\frac{3}{2} \lambda_{100}\left(\stackrel{k}{m}_{u} \stackrel{k}{m}_{u}-c_{0}\right), \\
& k_{u v}=3 \lambda_{111} \stackrel{k}{m_{u}} \stackrel{k}{m_{v}}, \quad u \neq v,
\end{aligned}
$$

where $u, v \in\{1,2,3\}$, and $c_{0}$ is a nondimensional stiffness parameter.

The unit magnitude, minimum energy directions $\underset{\mathbf{m}}{\mathbf{m}}$ are calculated from the inhomogeneous eigenvalue problem

$$
\begin{aligned}
(\mathbf{K}-\stackrel{k}{\gamma} \mathbf{I}) \stackrel{k}{\mathbf{m}}=K \stackrel{k}{\mathbf{c}}+\mu_{0} M_{s} \mathbf{H} \\
\stackrel{k}{\mathbf{m}} \cdot \stackrel{k}{\mathbf{m}}=1,
\end{aligned}
$$


where ${ }^{k}$ is the unknown Lagrange multiplier corresponding to the $k^{\text {th }}$ minimum energy direction, and $\mathbf{I}$ is the 3 by 3 identity matrix.

Letting $\mathbf{Q}$ be the orthogonal matrix containing the eigenvectors of $\mathbf{K}$ (with the eigenvalues $\lambda_{1}, \lambda_{2}$, and $\left.\lambda_{3}\right)$, Tari et al. [29] reported that

$$
\stackrel{k}{\mathbf{m}}=\mathbf{Q}\left[\begin{array}{ccc}
\frac{1}{\lambda_{1}-\underline{k}} & 0 & 0 \\
0 & \frac{1}{\lambda_{2}-\gamma} & 0 \\
0 & 0 & \frac{1}{\lambda_{3}-\gamma}
\end{array}\right] \mathbf{Q}^{\mathrm{T}}\left(K^{k} \stackrel{k}{\mathbf{c}}+\mu_{0} M_{s} \mathbf{H}\right)
$$

where $\stackrel{k}{\gamma}$ is obtained from the sixth order polynomial:

$$
\begin{array}{r}
\frac{k}{\gamma}{ }^{2}+2\left(\bar{\lambda}_{2}+\bar{\lambda}_{3}\right) \frac{k}{\gamma}{ }^{5}+\left(\bar{\lambda}_{2}^{2}+4 \bar{\lambda}_{2} \bar{\lambda}_{3}+\bar{\lambda}_{3}^{2}-\bar{Q}_{1}-\bar{Q}_{2}-\bar{Q}_{3}\right) \frac{k}{\gamma} 4 \\
+2\left(\bar{\lambda}_{2}^{2} \bar{\lambda}_{3}+\bar{\lambda}_{2} \bar{\lambda}_{3}^{2}-\bar{\lambda}_{2} \bar{Q}_{1}-\bar{\lambda}_{3} \bar{Q}_{1}-\bar{\lambda}_{3} \bar{Q}_{2}-\bar{\lambda}_{2} \bar{Q}_{3}\right) \frac{k}{\gamma} 3 \\
+\left(\bar{\lambda}_{2}^{2} \bar{\lambda}_{3}^{2}-\bar{\lambda}_{2}^{2} \bar{Q}_{1}-4 \bar{\lambda}_{2} \bar{\lambda}_{3} \bar{Q}_{1}-\bar{\lambda}_{3}^{2} \bar{Q}_{1}-\bar{\lambda}_{3}^{2} \bar{Q}_{2}-\bar{\lambda}_{2}^{2} \bar{Q}_{3}\right) \frac{k}{\gamma}{ }^{2} \\
-2 \bar{\lambda}_{2} \bar{\lambda}_{3} \bar{Q}_{1}\left(\bar{\lambda}_{2}+\bar{\lambda}_{3}\right) \frac{k}{\gamma}-\bar{\lambda}_{2}^{2} \bar{\lambda}_{3}^{2} \bar{Q}_{1}=0
\end{array}
$$

with $\frac{k}{\gamma}=\lambda_{1}-\stackrel{k}{\gamma}, \bar{\lambda}_{2}=\lambda_{2}-\lambda_{1}, \bar{\lambda}_{3}=\lambda_{3}-\lambda_{1}$, and $\left[\sqrt{\bar{Q}_{1}} ; \sqrt{\bar{Q}_{2}} ; \sqrt{\bar{Q}_{3}}\right]=\mathbf{Q}^{\mathrm{T}}\left(K^{k} \mathbf{c}+\mu_{0} M_{s} \mathbf{H}\right)$. When eq. (17) has multiple real solutions, the one that results in the lowest Gibbs free energy is selected.

\section{Derivative Terms for the Direct Model for Galfenol}

This section presents analytical derivations of the Jacobian and Hessian terms for the direct model given in the previous section for Galfenol. As before, the same indicial notation is adopted.

\subsection{Jacobian terms}

The Jacobian terms can be obtained from differentiating eqs. (11) and (12) with respect to $H_{p}$ and $T_{i}$ as 


$$
\begin{aligned}
\frac{\partial \mathbf{B}}{\partial H_{p}} & =\mu_{0} \stackrel{p}{\mathbf{e}}+\mu_{0} M_{s} \sum_{k= \pm 1}^{ \pm r / 2}\left(\frac{\partial \bar{\xi}_{\text {hys }}}{\partial H_{p}} \stackrel{k}{\mathbf{m}}+{\stackrel{k}{\xi_{h y s}}}_{\text {h }} \frac{\partial \stackrel{k}{\mathbf{m}}}{\partial H_{p}}\right) \\
\frac{\partial \mathbf{B}}{\partial T_{i}} & =\mu_{0} M_{s} \sum_{k= \pm 1}^{ \pm r / 2}\left(\frac{\partial \frac{k}{\bar{\xi}_{\text {hys }}}}{\partial T_{i}} \stackrel{k}{\mathbf{m}}+\frac{k}{\bar{\xi}_{\text {hys }}} \frac{\partial \stackrel{k}{\mathbf{m}}}{\partial T_{i}}\right)
\end{aligned}
$$

and

$$
\begin{aligned}
\frac{\partial \mathbf{S}}{\partial H_{p}} & =\sum_{k= \pm 1}^{ \pm r / 2} \frac{\partial \bar{\xi}_{\text {hys }}}{\partial H_{p}} \stackrel{k}{\boldsymbol{\lambda}}+\sum_{k= \pm 1}^{ \pm r / 2} \frac{k}{\bar{\xi}_{\text {hys }}} \frac{\partial \stackrel{k}{\boldsymbol{\lambda}}}{\partial H_{p}} \\
\frac{\partial \mathbf{S}}{\partial T_{i}} & =\mathbf{s} \mathbf{e}+\sum_{k= \pm 1}^{ \pm r / 2} \frac{\partial \bar{\xi}_{\text {hys }}}{\partial T_{i}} \stackrel{k}{\boldsymbol{\lambda}}+\sum_{k= \pm 1}^{ \pm r / 2} \frac{k}{\bar{\xi}_{\text {hys }}} \frac{\partial \stackrel{k}{\boldsymbol{\lambda}}}{\partial T_{i}}
\end{aligned}
$$

where $\stackrel{p}{\mathbf{e}}$ and $\stackrel{i}{\mathbf{e}}$ are, respectively, 3 - and 6-dimensional unit vectors with one as their $p^{\text {th }}$ and $i^{\text {th }}$ components.

The derivatives of the averaged hysteretic volume fractions occurring in eqs. (18) and (19) are found upon differentiating eq. (13) with respect to field and stress, respectively, and simplifying the results as

$$
\begin{aligned}
& \frac{\partial \bar{\xi}_{\text {hys }}}{\partial H_{p}}=\frac{\mu_{0}}{\Omega}{\frac{k}{\xi_{h y s}}}_{\left(M_{s} \stackrel{k}{m}_{p}-\bar{M}_{p}\right),} \\
& \frac{\partial \frac{k}{\xi}_{\text {hys }}}{\partial T_{i}}=\frac{1}{\Omega} \frac{k}{\bar{\xi}_{\text {hys }}}\left(\stackrel{k}{\lambda_{i}}-\bar{\lambda}_{i}\right) .
\end{aligned}
$$

The remaining derivative terms in eq. (18) can be obtained by differentiating the inhomogeneous eigenvalue problem (15) with respect to field and stress, and solving the resulting equations as

$$
\begin{aligned}
& \frac{\partial \stackrel{k}{\mathbf{m}}}{\partial H_{p}}=[\mathbf{K}-\stackrel{k}{\gamma} \mathbf{I}]^{-1}\left(\frac{\partial \stackrel{k}{\gamma}}{\partial H_{p}} \stackrel{k}{\mathbf{m}}+\mu_{0} M_{s} \stackrel{p}{\mathbf{e}}\right), \\
& \frac{\partial \stackrel{k}{\mathbf{m}}}{\partial T_{i}}=[\mathbf{K}-\stackrel{k}{\gamma} \mathbf{I}]^{-1}\left(\frac{\partial \stackrel{k}{\gamma}}{\partial T_{i}} \stackrel{k}{\mathbf{m}}-\frac{\partial \mathbf{K}}{\partial T_{i}} \stackrel{k}{\mathbf{m}}\right),
\end{aligned}
$$

where 


$$
\begin{aligned}
& \frac{\partial \stackrel{k}{\gamma}}{\partial H_{p}}=-\mu_{0} M_{s} \frac{\stackrel{p}{\mathbf{e}} \cdot[\mathbf{K}-\stackrel{k}{\gamma} \mathbf{I}]^{-1} \stackrel{k}{\mathbf{m}}}{\underset{\mathbf{m}}{\mathbf{m}} \cdot[\mathbf{K}-\stackrel{k}{\gamma} \mathbf{I}]^{-1} \stackrel{k}{\mathbf{m}}}, \\
& \frac{\partial \stackrel{k}{\gamma}}{\partial T_{i}}=\frac{\frac{\partial \mathbf{K}}{\partial T_{i}} \stackrel{k}{\mathbf{m}} \cdot[\mathbf{K}-\stackrel{k}{\gamma} \mathbf{I}]^{-1} \stackrel{k}{\mathbf{m}}}{\stackrel{k}{\mathbf{m}} \cdot[\mathbf{K}-\stackrel{k}{\gamma} \mathbf{I}]^{-1} \stackrel{k}{\mathbf{m}}}
\end{aligned}
$$

Following a simplification procedure, the foregoing results can be combined as

$$
\begin{aligned}
& \frac{\partial \stackrel{k}{\mathbf{m}}}{\partial H_{p}}=-\mu_{0} M_{s} \stackrel{k}{\Gamma} \stackrel{k}{\mathbf{m}} \times[[\mathbf{K}-\stackrel{k}{\gamma} \mathbf{I}](\stackrel{k}{\mathbf{m}} \times \stackrel{p}{\mathbf{e}})], \\
& \frac{\partial \stackrel{k}{\mathbf{m}}}{\partial T_{i}}=\stackrel{k}{\Gamma} \stackrel{k}{\mathbf{m}} \times\left[[\mathbf{K}-\stackrel{k}{\gamma} \mathbf{I}]\left(\stackrel{k}{\mathbf{m}} \times \frac{\partial \mathbf{K}}{\partial T_{i}} \stackrel{k}{\mathbf{m}}\right)\right],
\end{aligned}
$$

where " $\times$ " is the vector cross product operator, and making use of the matrix determinant operator "| |"

$$
\stackrel{k}{\Gamma}=\frac{|\mathbf{K}-\stackrel{k}{\gamma} \mathbf{I}|^{-1}}{\stackrel{k}{\mathbf{m}} \cdot[\mathbf{K}-\stackrel{k}{\gamma} \mathbf{I}]^{-1} \stackrel{k}{\mathbf{m}}}
$$

Finally, the remaining derivative terms in eq. (19) can be obtained by differentiating eq. (14) with respect to field and stress as

$$
\begin{aligned}
& \frac{\partial \stackrel{k}{\lambda_{u u}}}{\partial H_{p}}=3 \lambda_{100} \stackrel{k}{m_{u}} \frac{\partial \stackrel{k}{m_{u}}}{\partial H_{p}}, \\
& \frac{\partial \stackrel{k}{\lambda_{u v}}}{\partial H_{p}}=3 \lambda_{111}\left(\stackrel{k}{m_{u}} \frac{\partial \stackrel{k}{m}_{v}}{\partial H_{p}}+\stackrel{k}{m_{v}} \frac{\partial \stackrel{k}{m}_{u}}{\partial H_{p}}\right), \quad u \neq v, \\
& \frac{\partial \stackrel{k}{\lambda_{u u}}}{\partial T_{i}}=3 \lambda_{100} \stackrel{k}{m_{u}} \frac{\partial \stackrel{k}{m}_{u}}{\partial T_{i}}, \\
& \frac{\partial \stackrel{k}{\lambda_{u v}}}{\partial T_{i}}=3 \lambda_{111}\left(\mathfrak{k}_{u} \frac{\partial \stackrel{k}{m}_{v}}{\partial T_{i}}+\stackrel{k}{m_{v}} \frac{\partial \stackrel{k}{m}_{u}}{\partial T_{i}}\right), \quad u \neq v .
\end{aligned}
$$

\subsection{Hessian terms}

The Hessian terms can be obtained from differentiating eq. (18) with respect to $H_{p}$ and $T_{i}$ as

$$
\frac{\partial^{2} \mathbf{B}}{\partial H_{p} \partial H_{q}}=\mu_{0} M_{s} \sum_{k= \pm 1}^{ \pm r / 2}\left(\frac{\partial^{2} \bar{\xi}_{\text {hys }}}{\partial H_{p} \partial H_{q}} \stackrel{k}{\mathbf{m}}+\frac{\partial \frac{k}{\xi}_{h y s}}{\partial H_{p}} \frac{\partial \stackrel{k}{\mathbf{m}}}{\partial H_{q}}+\frac{k}{\xi_{h y s}} \frac{\partial^{2} \stackrel{k}{\mathbf{m}}}{\partial H_{p} \partial H_{q}}+\frac{\partial \bar{\xi}_{\text {hys }}}{\partial H_{q}} \frac{\partial \stackrel{k}{\mathbf{m}}}{\partial H_{p}}\right)
$$




$$
\frac{\partial^{2} \mathbf{B}}{\partial T_{i} \partial T_{j}}=\mu_{0} M_{s} \sum_{k= \pm 1}^{ \pm r / 2}\left(\frac{\partial^{2} \bar{\xi}_{\text {hys }}}{\partial T_{i} \partial T_{j}} \stackrel{\underset{\mathbf{m}}{\mathbf{m}}}{+}+\frac{\partial \bar{\xi}_{\text {hys }}}{\partial T_{i}} \frac{\partial \stackrel{k}{\mathbf{m}}}{\partial T_{j}}+\frac{k}{\bar{\xi}_{h y s}} \frac{\partial^{2} \stackrel{k}{\mathbf{m}}}{\partial T_{i} \partial T_{j}}+\frac{\partial \bar{\xi}_{\text {hys }}}{\partial T_{j}} \frac{\partial \stackrel{k}{\mathbf{m}}}{\partial T_{i}}\right)
$$

and

$$
\frac{\partial^{2} \mathbf{B}}{\partial H_{p} \partial T_{i}}=\mu_{0} M_{s} \sum_{k= \pm 1}^{ \pm r / 2}\left(\frac{\partial^{2} \bar{\xi}_{\text {hys }}}{\partial H_{p} \partial T_{i}} \stackrel{k}{\mathbf{m}}+\frac{\partial \bar{\xi}_{\text {hys }}}{\partial H_{p}} \frac{\partial \stackrel{k}{\mathbf{m}}}{\partial T_{i}}+\frac{k}{\bar{\xi}_{h y s}} \frac{\partial^{2} \stackrel{k}{\mathbf{m}}}{\partial H_{p} \partial T_{i}}+\frac{\partial \bar{\xi}_{h y s}}{\partial T_{i}} \frac{\partial \stackrel{k}{\mathbf{m}}}{\partial H_{p}}\right)
$$

and differentiating eq. (19) with respect to $H_{p}$ and $T_{i}$ as

$$
\begin{aligned}
& \frac{\partial^{2} \mathbf{S}}{\partial H_{p} \partial H_{q}}=\sum_{k= \pm 1}^{ \pm r / 2}\left(\frac{\partial^{2} \bar{\xi}_{h y s}}{\partial H_{p} \partial H_{q}} \stackrel{k}{\lambda}+\frac{\partial \bar{\xi}_{\text {hys }}}{\partial H_{p}} \frac{\partial \stackrel{k}{\lambda}}{\partial H_{q}}+\frac{k}{\bar{\xi}_{h y s}} \frac{\partial^{2} \stackrel{k}{\lambda}}{\partial H_{p} \partial H_{q}}+\frac{\partial{\frac{k}{\xi_{h y s}}}_{\partial H_{q}}}{\partial H_{p}}\right) \\
& \frac{\partial^{2} \mathbf{S}}{\partial T_{i} \partial T_{j}}=\sum_{k= \pm 1}^{ \pm r / 2}\left(\frac{\partial^{2} \bar{\xi}_{\text {hys }}}{\partial T_{i} \partial T_{j}} \stackrel{k}{\boldsymbol{\lambda}}+\frac{\partial \frac{k}{\bar{\xi}_{h y s}}}{\partial T_{i}} \frac{\partial \stackrel{k}{\lambda}}{\partial T_{j}}+\frac{k}{\xi}_{h y s} \frac{\partial^{2} \stackrel{k}{\lambda}}{\partial T_{i} \partial T_{j}}+\frac{\partial \bar{\xi}_{\text {hys }}}{\partial T_{j}} \frac{\partial \stackrel{k}{\lambda}}{\partial T_{i}}\right)
\end{aligned}
$$

and

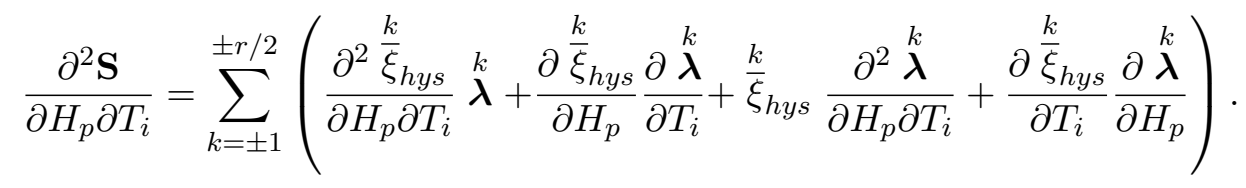

The second order derivatives of the averaged hysteretic volume fractions in the foregoing equations may be obtained from differentiating eq. (20) with respect to field and stress as

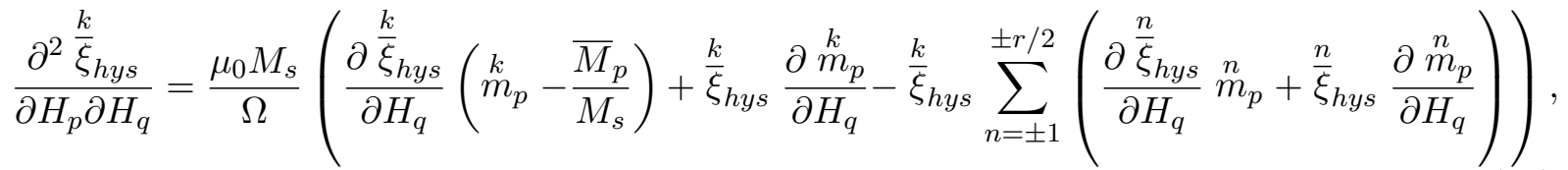

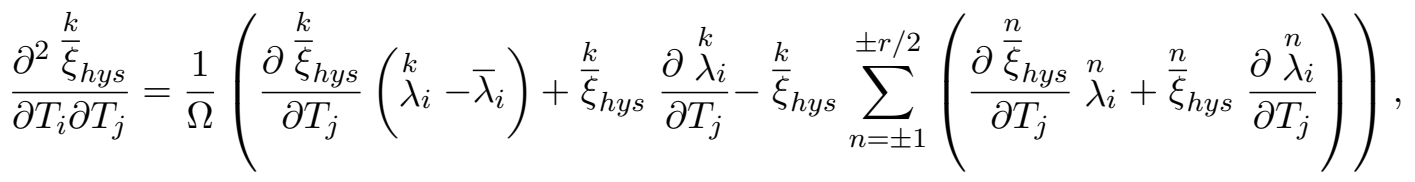

and 


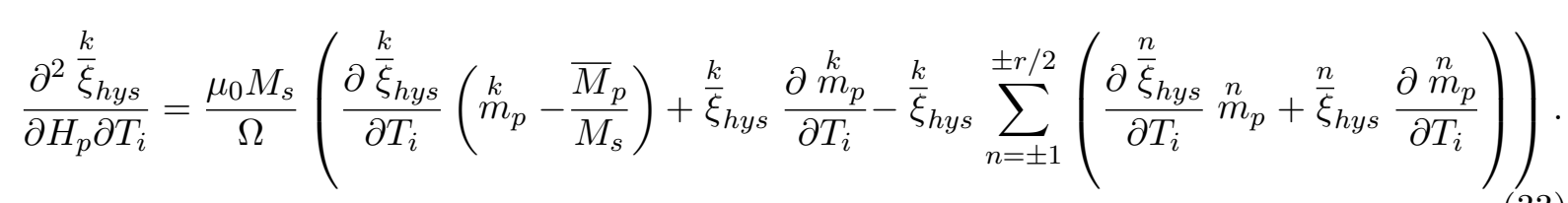

To simplify the remaining second order derivative terms in eqs. (25)-(27), we differentiate the inhomogeneous eigenvalue problem (15) twice with respect to field and stress, and solve the resulting equations for the emerging intermediate unknowns. After a simplification procedure, we finally have

$$
\begin{aligned}
& \frac{\partial^{2} \stackrel{k}{\mathbf{m}}}{\partial H_{p} \partial H_{q}}=-\stackrel{k}{\Gamma}|\mathbf{K}-\stackrel{k}{\gamma} \mathbf{I}|\left(\frac{\partial \stackrel{k}{\mathbf{m}}}{\partial H_{p}} \cdot \frac{\partial \stackrel{k}{\mathbf{m}}}{\partial H_{q}}\right)[\mathbf{K}-\stackrel{k}{\gamma} \mathbf{I}]^{-1} \stackrel{k}{\mathbf{m}} \\
&-\stackrel{k}{\Gamma} \stackrel{k}{\mathbf{m}} \times[\mathbf{K}-\stackrel{k}{\gamma} \mathbf{I}]\left(\stackrel{k}{\mathbf{m}} \times\left[\frac{\partial \stackrel{k}{\gamma}}{\partial H_{p}} \frac{\partial \stackrel{k}{\mathbf{m}}}{\partial H_{q}}+\frac{\partial \stackrel{k}{\gamma}}{\partial H_{q}} \frac{\partial \stackrel{k}{\mathbf{m}}}{\partial H_{p}}\right]\right), \\
& \frac{\partial^{2} \stackrel{k}{\mathbf{m}}}{\partial T_{i} \partial T_{j}}=-\stackrel{k}{\Gamma}|\mathbf{K}-\stackrel{k}{\gamma} \mathbf{I}|\left(\frac{\partial \stackrel{k}{\mathbf{m}}}{\partial T_{i}} \cdot \frac{\partial \stackrel{k}{\mathbf{m}}}{\partial T_{j}}\right)[\mathbf{K}-\stackrel{k}{\gamma} \mathbf{I}]^{-1} \stackrel{k}{\mathbf{m}}+\stackrel{k}{\Gamma} \stackrel{k}{\mathbf{m}} \times \\
& \\
& {[\mathbf{K}-\stackrel{k}{\gamma} \mathbf{I}]\left(\underset{k}{\mathbf{m}} \times\left[\left[\frac{\partial \mathbf{K}}{\partial T_{i}}-\frac{\partial \stackrel{k}{\gamma}}{\partial T_{i}} \mathbf{I}\right] \frac{\partial \stackrel{k}{\mathbf{m}}}{\partial T_{j}}+\left[\frac{\partial \mathbf{K}}{\partial T_{j}}-\frac{\partial \stackrel{k}{\gamma}}{\partial T_{j}} \mathbf{I}\right] \frac{\partial \stackrel{k}{\mathbf{m}}}{\partial T_{i}}\right]\right), }
\end{aligned}
$$

and

$$
\begin{aligned}
\frac{\partial^{2} \stackrel{k}{\mathbf{m}}}{\partial H_{p} \partial T_{i}}= & -\stackrel{k}{\Gamma}|\mathbf{K}-\stackrel{k}{\gamma} \mathbf{I}|\left(\frac{\partial \stackrel{k}{\mathbf{m}}}{\partial H_{p}} \cdot \frac{\partial \stackrel{k}{\mathbf{m}}}{\partial T_{i}}\right)[\mathbf{K}-\stackrel{k}{\gamma} \mathbf{I}]^{-1} \stackrel{k}{\mathbf{m}} \\
& +\stackrel{k}{\Gamma} \stackrel{k}{\mathbf{m}} \times[\mathbf{K}-\stackrel{k}{\gamma} \mathbf{I}]\left(\stackrel{k}{\mathbf{m}} \times\left[\left[\frac{\partial \mathbf{K}}{\partial T_{i}}-\frac{\partial \stackrel{k}{\gamma}}{\partial T_{i}} \mathbf{I}\right] \frac{\partial \stackrel{k}{\mathbf{m}}}{\partial H_{p}}-\frac{\partial \stackrel{k}{\gamma}}{\partial H_{p}} \frac{\partial \stackrel{k}{\mathbf{m}}}{\partial T_{i}}\right]\right)
\end{aligned}
$$

Finally, the remaining unknown derivative terms in eqs. (28)-(30) are obtained from differentiating eq. (24) with respect to field and stress as

$$
\begin{aligned}
\frac{\partial^{2} \stackrel{k}{\lambda}{ }_{u u}}{\partial H_{p} \partial H_{q}} & =3 \lambda_{100}\left(\stackrel{k}{m_{u}} \frac{\partial^{2} \stackrel{k}{m}_{u}}{\partial H_{p} \partial H_{q}}+\frac{\partial \stackrel{k}{m_{u}}}{\partial H_{p}} \frac{\partial \stackrel{k}{m_{u}}}{\partial H_{q}}\right) \\
\frac{\partial^{2} \stackrel{k}{\lambda}}{\partial H_{p} \partial H_{q}} & =3 \lambda_{111}\left(\stackrel{k}{m_{u}} \frac{\partial^{2} \stackrel{k}{m}_{v}}{\partial H_{p} \partial H_{q}}+\frac{\partial \stackrel{k}{m}_{u}}{\partial H_{p}} \frac{\partial \stackrel{k}{m_{v}}}{\partial H_{q}}+\stackrel{k}{m_{v}} \frac{\partial^{2} \stackrel{k}{m}_{u}}{\partial H_{p} \partial H_{q}}+\frac{\partial \stackrel{k}{m_{v}}}{\partial H_{p}} \frac{\partial m_{u}}{\partial H_{q}}\right), \quad u \neq v
\end{aligned}
$$




$$
\begin{aligned}
& \frac{\partial^{2} \stackrel{k}{\lambda_{u u}}}{\partial T_{i} \partial T_{j}}=3 \lambda_{100}\left(\stackrel{k}{m_{u}} \frac{\partial^{2} \stackrel{k}{m}_{u}}{\partial T_{i} \partial T_{j}}+\frac{\partial \stackrel{k}{m_{u}}}{\partial T_{i}} \frac{\partial \stackrel{k}{m}_{u}}{\partial T_{j}}\right), \\
& \frac{\partial^{2} \stackrel{k}{\lambda_{u v}}}{\partial T_{i} \partial T_{j}}=3 \lambda_{111}\left(\stackrel{k}{m_{u}} \frac{\partial^{2} \stackrel{k}{m}_{v}}{\partial T_{i} \partial T_{j}}+\frac{\partial \stackrel{k}{m_{u}}}{\partial T_{i}} \frac{\partial \stackrel{k}{m_{v}}}{\partial T_{j}}+\stackrel{k}{m_{v}} \frac{\partial^{2} \stackrel{k}{m}_{u}}{\partial T_{i} \partial T_{j}}+\frac{\partial \stackrel{k}{m_{v}}}{\partial T_{i}} \frac{\partial \stackrel{k}{m_{u}}}{\partial T_{j}}\right), \quad u \neq v,
\end{aligned}
$$

and

$$
\begin{aligned}
& \frac{\partial^{2} \stackrel{k}{\lambda_{u u}}}{\partial H_{p} \partial T_{i}}=3 \lambda_{100}\left(\stackrel{k}{m_{u}} \frac{\partial^{2} \stackrel{k}{m_{u}}}{\partial H_{p} \partial T_{i}}+\frac{\partial \stackrel{k}{m_{u}}}{\partial H_{p}} \frac{\partial \stackrel{k}{m_{u}}}{\partial T_{i}}\right) \\
& \frac{\partial^{2} \stackrel{k}{\lambda}_{u v}}{\partial H_{p} \partial T_{i}}=3 \lambda_{111}\left(\stackrel{k}{m_{u}} \frac{\partial^{2} \stackrel{k}{m}_{v}}{\partial H_{p} \partial T_{i}}+\frac{\partial \stackrel{k}{m_{u}}}{\partial H_{p}} \frac{\partial \stackrel{k}{m}_{v}}{\partial T_{i}}+\stackrel{k}{m_{v}} \frac{\partial^{2} \stackrel{k}{m_{u}}}{\partial H_{p} \partial T_{i}}+\frac{\partial \stackrel{k}{m_{v}}}{\partial H_{p}} \frac{\partial m_{u}^{k}}{\partial T_{i}}\right), \quad u \neq v .
\end{aligned}
$$

\section{Inverse Model Performance for 1D Input Data}

We benchmark the performance of the proposed inverse model for special and general input data. The model's insensitivity to special input data is assessed in this section with 1D input data, and its robustness for general input data is demonstrated in the next section with a fully coupled, 3D simulation of a magnetostrictive composite plate actuator.

We compare the convergence rate of the proposed inverse model with that of the quasiNewton based inverse model presented by Deng and Dapino [28]. The latter does not offer the continuation feature, therefore, for a fair comparison, we disable continuation in the proposed model by setting the continuation parameter $\tau$ to one. To generate comparative data, 1D magnetic field and stress spaces are specified, with the constraint that the sampled stress tensor must have a von Mises stress smaller than $500 \mathrm{MPa}$, which is a rough estimate of the ultimate strength of Galfenol. Each space is then discretized and fed to the direct model, given in Section 3, to produce magnetic induction and strain spaces. To benchmark the two inverse models, these datasets are fed to each inverse model to see whether the original magnetic field and stress inputs are returned up to a tolerance of $10^{-9}$.

As for the direct model parameters, Evans and Dapino [25] collected magnetic induction and strain measurements, for uniaxial actuation and sensing, of textured Fe81.5 Ga18.5 grown in $\langle 100\rangle$ along the rod axis with the Free Stand Zone Melt method (FSZM) at Etrema Products Inc. For this dataset, Tari et al. [29] reported the direct model parameters, which are tabulated in Table 1. 
(a)

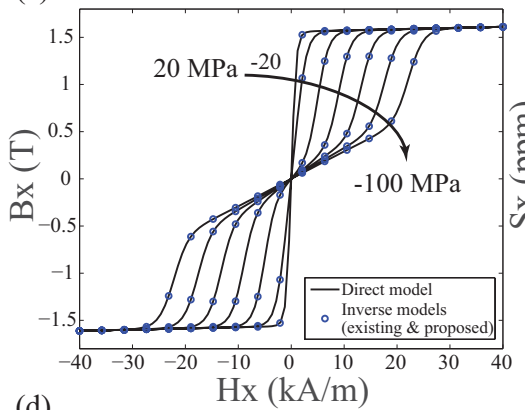

(d)

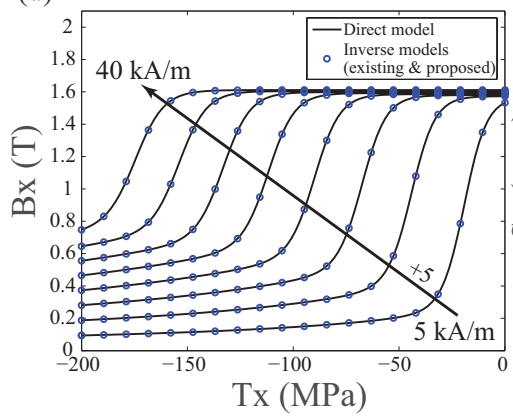

(b)

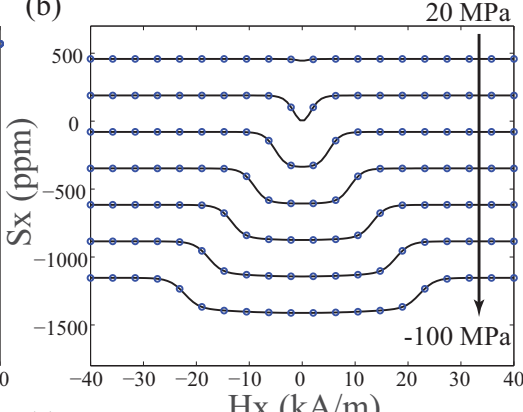

(e)

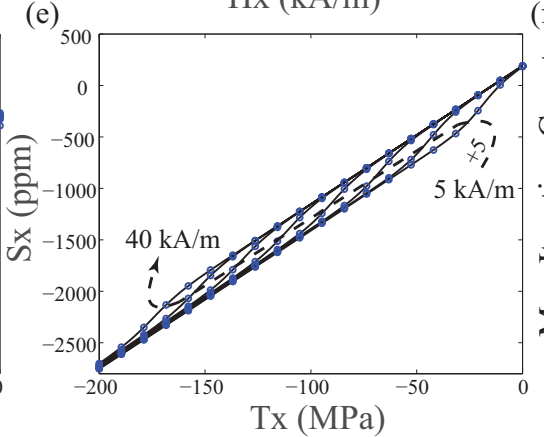

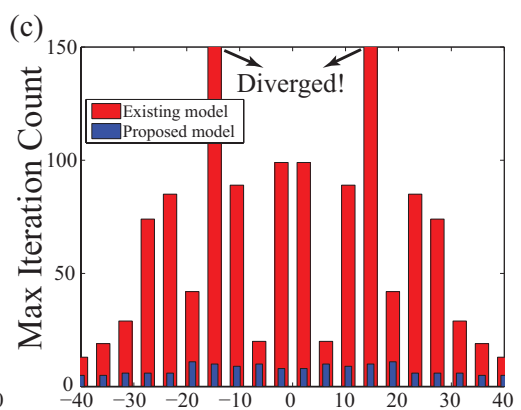

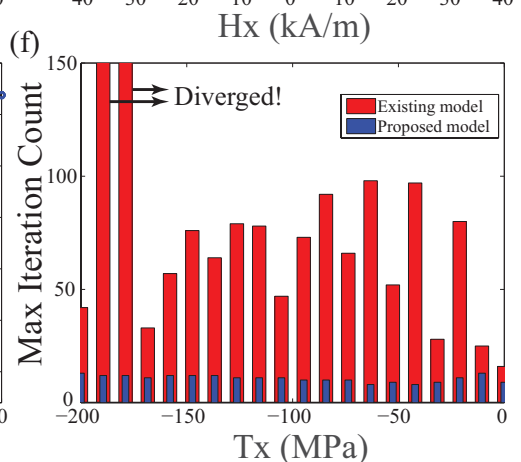

Figure 1: Direct and inverse model simulations for $\langle 100\rangle \mathrm{Fe}_{81.5} \mathrm{Ga}_{18.5}$ grown with FSZM for 1D (a), (b), and (c) actuation; and (d), (e), and (f) sensing. The existing inverse model is that of Deng and Dapino [28], and also $\mathrm{H}_{\mathrm{x}}$, $\mathrm{T}_{\mathrm{x}}, \mathrm{B}_{\mathrm{x}}$ and $\mathrm{S}_{\mathrm{x}}$ are the $\mathrm{x}$-component of the magnetic field, stress, magnetic induction and strain, respectively.

Table 1: Parameters for the direct model, given in Section 3, for $\langle 100\rangle$ Fe $_{81.5}$ Ga $_{18.5}$ grown with FSZM (Tari et al. [29]).

\begin{tabular}{|c|cccc|}
\hline Par. & $M_{s}(\mathrm{kA} / \mathrm{m})$ & $E(\mathrm{GPa})$ & $\lambda_{100}(\mathrm{ppm})$ & $\lambda_{111}(\mathrm{ppm})$ \\
\hline Value & 1242.20 & 74.49 & 172.31 & 0.00 \\
\hline \hline Par. & $c_{0}$ & $K\left(\mathrm{~kJ} / \mathrm{m}^{3}\right)$ & $K_{100}\left(\mathrm{~J} / \mathrm{m}^{3}\right)$ & $\Omega(\mathrm{J})$ \\
\hline Value & 0.38 & 35.58 & 412.18 & 1330.00 \\
\hline
\end{tabular}

Figure 1 illustrates the direct and inverse model simulations for 1D magnetic field and stress inputs. This follows the 1D uniaxial actuation and sensing experiment conducted by Evans and Dapino [25], in which a magnetostrictive rod is exposed to load and magnetic field along its main axis, and the magnetic induction and strain are measured. The solid curves in subfigures (a), (b), (d), and (e) represent the direct model simulations for actuation and sensing cases. As explained above, equally spaced points are chosen on these curves, and are fed to the two inverse models, i.e. the proposed model and the existing model by Deng and Dapino [28].

When the stress is held constant but the magnetic field is changing in one dimension, the inversion procedure must be independent of the sign of the field inputs. This is demonstrated in subfigure 1 (c), as the iteration counts for both inversion models are symmetric about the origin. 
In addition, subfigures 1 (c) and (f) depict the maximum number of iterations that the two inverse models take, respectively, at constant stress and magnetic field states. Both the existing and proposed inverse models are able to solve the inverse magnetomechanical problem effectively. Nonetheless, the existing approach has a few failures for this 1D case. Furthermore, the proposed approach converges in significantly fewer iterations than the existing approach. This faster rate of convergence is expected, as the proposed model employs exact derivative terms, while the existing approach employs approximate terms, development of which is a premise of the quasiNewton method.

\subsection{Choice of the iterative step size $\alpha$}

For both the quasi-Newton method and the damped Newton method, the step size $\alpha$, present for example in eq. (6), must be calculated at each iteration. There is a variety of line search algorithms for this purpose, but they fall into two major categories: exact and approximate. For the simulations, an exact line search based on Golden Section Search (see, for example, Ref. [36]) is used. However, an approximate line search is often sufficient, and can save much computational time. This will be the subject of future work.

\subsection{Choice of start solution}

Start solutions have a major role in the success of iterative techniques, such as quasi- or damped Newton methods. Even if chosen within the convergence zone, an ill-conditioned start solution (i.e., one with a high condition number) may lead to a long runtime. As shown in previous sections, the exact derivative terms, even if compact, are unwieldy, and computationally expensive to evaluate. Even if they lead to minimal number of iterations, they are most efficient when used for local rather than global convergence. Therefore, it is more efficient to carry on a preprocessing step in which a less robust but faster approach such as quasi-Newton method is used to generate a reasonable approximate solution. Then, feeding this solution as a start solution to the main algorithm would save computational time. Alternatively, one may use continuation, which is self-constructive, as it automatically generates a reasonably well-conditioned start solution through solving a series of intermediate subproblems.

For the simulations, zero start solutions are used. However, the inverse model is devised for use in a finite-element framework. In such a case, the system state is known at some instant, and this state is a good candidate as a start solution for the next state. This feature is useful 
for control applications. In what follows, the integration of the proposed inverse model within a finite-element framework is demonstrated.

\section{Fully Coupled Simulation of a Magnetostrictive Composite Plate Actuator}

The Galfenol-aluminum composite actuator shown in Figure 2 is the schematic view of the sample utilized in the experimental set-up by Santapuri et al. [37].

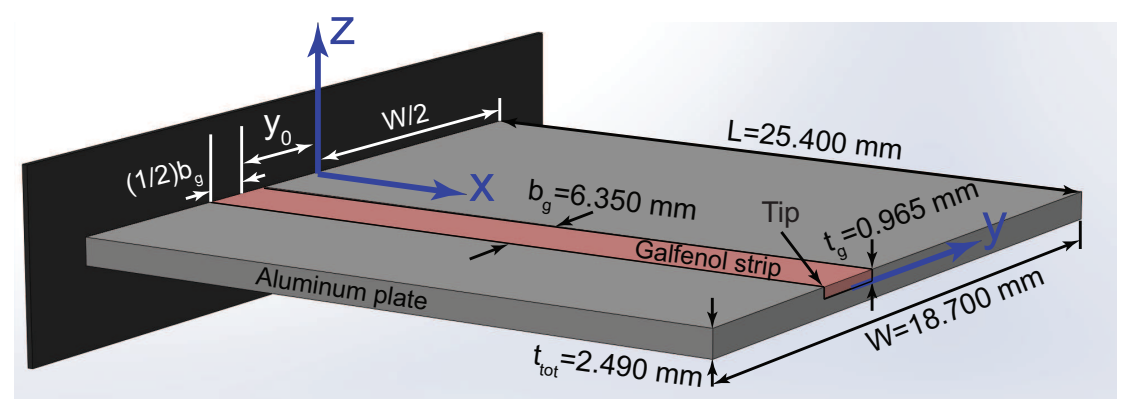

Figure 2: Schematic of a Galfenol-aluminium composite structure. The $x-y$ plane of the coordinate system is coincident with the bottom plane of the Galfenol strip, and the $x-z$ plane is a plane of symmetry $\left(y_{0}=0\right)$.

In the experiment, the plate was excited using 1D magnetic fields along the Galfenol length, and cauchy boundary conditions were imposed at the clamped edge. Input alternating and bias magnetic fields of different amplitudes and frequencies (ranging from $0.1 \mathrm{~Hz}$ to $250 \mathrm{~Hz}$ ) were generated using a conductive coil, and the displacement data at the tip of the Galfenol patch (labeled in Figure 2) was collected. Tari et al. [30] recently presented a reduced 2D model for this composite plate with the assumption that the magnetic field distribution throughout the Galfenol volume is approximately uniform for the small deformations, thus they assumed that the magnetic field is known. However, we lift this assumption in this work, and simulate this composite actuator in full 3D.

\subsection{The Governing Equations}

The governing equations describe structural behavior, electromagnetic behavior, and material constitutive behavior. Following Tari et al. [30], the structural behavior is described by the weak form of the 3D Navier's equation as

$$
\int_{V_{t o t}}\left[\rho \frac{\partial^{2} \mathbf{u}}{\partial t^{2}} \cdot \delta \mathbf{u}+c \frac{\partial \mathbf{u}}{\partial t} \cdot \delta \mathbf{u}+\mathbf{T} \cdot \delta \mathbf{S}\right] d V=\int_{\partial V_{t o t}} \mathbf{t} \cdot \delta \mathbf{u} d \partial V+\int_{V_{t o t}} \mathbf{f}_{B} \cdot \delta \mathbf{u} d V
$$


where $t$ is the time, $\rho$ is the density, $c$ represents the damping coefficient, and $\mathbf{T}$ and $\mathbf{f}_{B}$ denote, respectively, the stress tensor and external body force acting on the mechanical domain $V_{\text {tot }}$; the traction vector $\mathbf{t}$ acts on the boundary $\partial V_{\text {tot }}$; and $\mathbf{S}$ and $\mathbf{u}$ represent, respectively, the strain tensor and displacement vector at each point in the domain $V_{t o t}$ with the fact that

$$
\mathbf{S}=\frac{1}{2}\left(\nabla \mathbf{u}+\nabla \mathbf{u}^{\mathrm{T}}\right)
$$

Following Chakrabarti and Dapino [27], the magnetic behavior is described by the weak form of a modified form of Ampere's law as

$$
\int_{V_{B}}\left[\mathbf{H} \cdot \delta \mathbf{B}+\sigma \frac{\partial \mathbf{A}}{\partial t} \cdot \delta \mathbf{A}\right] d V=\int_{\partial V_{B}}(\mathbf{H} \times \mathbf{n}) \cdot \delta \mathbf{A} d \partial V+\int_{V_{B}} \mathbf{J}_{s} \cdot \delta \mathbf{A} d V
$$

where $V_{B}$ is the electromagnetic domain and $\partial V_{B}$ is its boundary with the outer normal $\mathbf{n} ; \mathbf{J}_{s}$ is the source current density (applied through a coil) and $\sigma$ is the conductivity of the material; and finally, $\mathbf{H}$ is the magnetic field vector, $\mathbf{B}$ is the magnetic flux density vector, and $\mathbf{A}$ is the magnetic potential vector defined by

$$
\mathbf{B}=\nabla \times \mathbf{A}
$$

Finally, the passive material behavior is governed by the constitutive Hooke's law

$$
\mathbf{T}_{p}=\mathbf{C}_{p} \cdot \mathbf{S}_{p}
$$

where the subscript $p$ signifies the passive domain, and $\mathbf{C}_{p}$ is the second rank modulus tensor.

\subsection{Solution Approach}

As employed by Tari et al. [30], the material parameters for aluminum are $E=69$ GPa, $\nu=0.3$, and $\rho=2,700 \mathrm{~kg} / \mathrm{m}^{3}$; and those for Galfenol are tabulated in Table 2.

Table 2: Galfenol parameters from Tari et al. [30].

\begin{tabular}{|c|cccccc|}
\hline Par. & $M_{s}(\mathrm{kA} / \mathrm{m})$ & $E(\mathrm{GPa})$ & $\lambda_{100}(\mathrm{ppm})$ & $\lambda_{111}(\mathrm{ppm})$ & $c_{0}$ & $\rho\left(\mathrm{kg} / \mathrm{m}^{3}\right)$ \\
\hline Value & $1,273.24$ & 74.50 & 173.33 & -6.67 & 0.33 & 7870.00 \\
\hline \hline Par. & $K\left(\mathrm{~kJ} / \mathrm{m}^{3}\right)$ & $K_{100}\left(\mathrm{~J} / \mathrm{m}^{3}\right)$ & $\Omega(\mathrm{J})$ & $G(\mathrm{GPa})$ & $\nu$ & $c(\mathrm{~N} . \mathrm{s} / \mathrm{m})$ \\
\hline Value & 30.00 & -250.00 & $1,200.00$ & 120.00 & 0.30 & 0.10 \\
\hline
\end{tabular}

The equations given in the foregoing subsection are implemented in finite-element software COMSOL Multiphysics (version 4.4), and the constitutive model for Galfenol, given in Section 3, 
together with the inversion scheme developed in Section 2 are coded as MATLAB m-files and supplied to COMSOL. In particular, the weighting factors are taken as $w_{b}=1, w_{s}=10^{6}$, $w_{h}=10^{3}$, and $w_{t}=10^{6}$. Depicted in Figure 3, the actuator consists of the plate, a drive coil, and air volume sufficiently large so that the magnetic potential is negligible at the outer boundaries. The coil is the COMSOL's multi-turn circular coil with 800 turns and wire crosssectional area of $10^{-6} \mathrm{~m}^{2}$. In addition, user-controlled mesh is used: general physics for the plate, and free tetrahedral for the remaining constituents, so that the system attained total of 155,607 degrees of freedom. A harmonic current of the form $I(t)=I_{0} \sin (2 \pi \omega t)$ A is given as input to the coil, which creates magnetic field mainly along the plate length. The composite plate's behavior is simulated using COMSOL's BDF time-dependent solver for different values of input current amplitude $I_{0}$ and frequency $\omega$. As demonstrated in the following subsections, the model is convergent for the entire simulation cases.

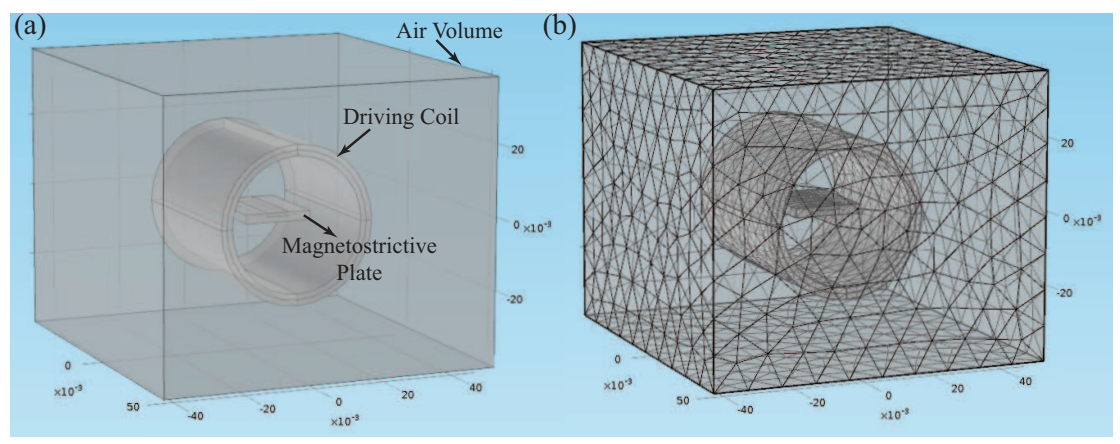

Figure 3: Magnetostrictive plate actuator setup in COMSOL: (a) geometry, and (b) mesh.

\subsection{Simulation Results}

Figure 4 illustrates the Galfenol tip displacement curves obtained from the simulations for the input current amplitude of $I_{0}=0.8 \mathrm{~A}$ and frequency of $\omega=50 \mathrm{~Hz}$ for two periods. As expected, there is negligible displacement in the y direction, while maximum displacement is observed in the $\mathrm{z}$ direction. Also, there is a positive displacement in the $\mathrm{x}$ direction, while a negative displacement in the $\mathrm{z}$ direction, as the Galfenol strip elongates and bends the plate downward.

Figure 5 depicts the obtained magnetic flux density and magnetic field simulation data for the running case. Even though the driving coil creates an external magnetic field predominantly along the $\mathrm{x}$ direction, the magnetic field and flux density components along all of the three $\mathrm{x}$, $\mathrm{y}$, and $\mathrm{z}$ axes inside Galfenol are at present. This is due to the stress effects in response to the Galfenol elongation, and also the boundary conditions imposed at the interface between the 
Galfenol strip and aluminum plate. In addition, the $\mathrm{z}$ components of the magnetic field and flux density shown in subfigure 5 (c) are linearly dependent. This was expected as, the Galfenol strip is rather thin in the $\mathrm{z}$ direction compared to the other directions. This linear dependence paves the way for model reduction and gaining computational efficiency in the future.

\subsubsection{Effect of Change of Input Current Amplitude}

Figure 6 shows the obtained displacement, magnetic flux density, and magnetic field simulation data for the input current amplitudes of $I_{0} \in\{0.6,0.8,1.0\}$ A and frequency of $\omega=50 \mathrm{~Hz}$ for two periods. As shown in subfigure 6 (c), Galfenol tip's displacement in the y direction, as expected, is zero and independent of the current amplitude. However, an increase in the current amplitude does increase the deflection in the remaining directions. For instance, Galfenol tip's maximum absolute displacement is $(0.081 ; 0 ; 0.493) \mu \mathrm{m}$ for $I_{0}=0.6 \mathrm{~A}$, and increases $84 \%$ and $63 \%$ for the two consecutive $0.2 \mathrm{~A}$ increase in the current magnitude, i.e. $I_{0}=0.8 \mathrm{~A}$ and $I_{0}=1.0 \mathrm{~A}$, respectively. Likewise, an increase in the current magnitude increases magnetic flux density. Shown in subfigures 6 (b), (d), and (f), the maximum magnetic flux density obtained at the Galfenol tip is $\mathbf{B}=(0.062 ; 0.058 ; 0.051) \mathrm{T}$, which increases $33 \%$ and $24 \%$ for the same aforementioned increases in the current magnitude.

\subsubsection{Effect of Change of Input Current Frequency}

Figure 7 illustrates the obtained displacement, magnetic flux density, and magnetic field simulation data for the input current frequencies of $\omega \in\{50,100,150\} \mathrm{Hz}$ and amplitude of $I_{0}=0.8 \mathrm{~A}$ for two periods. Similar to current amplitude, Galfenol tip's displacement in the y direction is zero and independent of the current frequency. On the other hand, while an increase in the current amplitude noticeably increases the $\mathrm{x}$ and $\mathrm{z}$ deflection components, an increase in the current frequency only marginally increases these deflection components but substantially intensifies hysteresis, which is signified by the enclosed area in each diagram.

The input current frequency has a similar effect on magnetic flux density; As shown in subfigures 7 (b) and (d), an increase in the current frequency increases hysteresis in the $\mathrm{x}$ and y magnetic flux density components. Nevertheless, the z component of magnetic flux density is rather independent of the current frequency. This is due to the slenderness of the Galfenol strip in the $\mathrm{z}$ direction. 


\section{Conclusions}

A fully coupled inverse model for arbitrary smart materials was presented. The model requirement is a continuous and second order differentiable direct model for any chosen smart material. The approach is globally convergent, which makes it ideal for use in finite-element frameworks. The premise of the existing iterative system models is to constitute recursive correction formulae based on first order approximations of some specified error functions. However, to achieve a faster convergence rate, we formulated the problem in an optimization framework through defining a novel scalar error function, and took second order approximations into ac-

count. A continuation approach was then developed to achieve global convergence for arbitrary input parameters.

Magnetostrictive Galfenol was chosen to illustrate the effectiveness of the inverse model, and fully compact analytical derivations of the Jacobian and Hessian matrices were presented for an existing Galfenol model. The convergence rate of the proposed approach was found to be superior to that of an existing inverse model, which is based on quasi-Newton method. An efficient line search algorithm and a carefully developed start solution are needed to gain further efficiency. As for the latter, start solutions are obviated when using continuation, which generates them automatically. Alternatively, when using the model in a finite-element framework, the current system state can be used as a reasonable candidate for the start solution.

In addition, the inverse model's robustness was successfully benchmarked through integrating the model into a finite-element framework to simulate an aluminum-Galfenol composite plate actuator. In contrast to an earlier work, we did not make any assumption regarding the distribution of magnetic field inside the magnetostrictive material, and rather conducted a full 3D, fully coupled simulation.

\section{Acknowledgment}

The authors acknowledge the financial support from the member organizations of the Smart Vehicle Concepts Center, a National Science Foundation Industry/University Cooperative Research Center established under grant IIP-1238286. Dr. Sushma Santapuri's assistance in setting up the plate model in COMSOL is acknowledged. 


\section{References}

[1] R. C. Smith, Smart material systems: model development, vol. 32, Society for Industrial and Applied Mathematics, 2005.

[2] D. J. Leo, Engineering analysis of smart material systems, Wiley InterScience, Wiley, 2007.

[3] W. G. Cady, Piezoelectricity: an introduction to the theory and applications of electromechanical phenomena in crystals, Dover New York, 1964.

[4] A. P. Stebner, L. C. Brinson, Explicit finite element implementation of an improved three dimensional constitutive model for shape memory alloys, Computer Methods in Applied Mechanics and Engineering 257 (2013) 17-35.

[5] C. Cisse, W. Zaki, T. B. Zineb, A review of constitutive models and modeling techniques for shape memory alloys, International Journal of Plasticity 76 (2016) 244-284.

[6] M.-A. Keip, P. Steinmann, J. Schröder, Two-scale computational homogenization of electroelasticity at finite strains, Computer Methods in Applied Mechanics and Engineering 278 (2014) 62-79.

[7] C. Linder, X. Zhang, Three-dimensional finite elements with embedded strong discontinuities to model failure in electromechanical coupled materials, Computer Methods in Applied Mechanics and Engineering 273 (2014) 143-160.

[8] W. Zheng, F. Zhang, Adaptive finite element frequency domain method for eddy current problems, Computer Methods in Applied Mechanics and Engineering 197 (13-16) (2008) $1233-1241$.

[9] C. Greif, D. Li, D. Schötzau, X. Wei, A mixed finite element method with exactly divergence-free velocities for incompressible magnetohydrodynamics, Computer Methods in Applied Mechanics and Engineering 199 (45-48) (2010) 2840-2855.

[10] G. Caloz, M. Dauge, E. Faou, V. Péron, On the influence of the geometry on skin effect in electromagnetism, Computer Methods in Applied Mechanics and Engineering 200 (9-12) (2011) 1053-1068. 
[11] X. Dong, Y. He, Y. Zhang, Convergence analysis of three finite element iterative methods for the 2D/3D stationary incompressible magnetohydrodynamics, Computer Methods in Applied Mechanics and Engineering 276 (2014) 287-311.

[12] S. Chakrabarti, M. J. Dapino, Fully coupled discrete energy-averaged model for Terfenol-D, Journal of Applied Physics 111 (5).

[13] R. A. Kellogg, Development and modeling of iron-gallium alloys, Ph.D. Dissertation, Iowa State University, 2003.

[14] L. Landau, E. Lifshitz, On the theory of the dispersion of magnetic permeability in ferromagnetic bodies, Physikalische Zeitschrift der Sowjetunion 8 (1935) 153.

[15] X.-P. Wang, C. J. García-Cervera, W. E, A Gauss-Seidel projection method for micromagnetics simulations, Journal of Computational Physics 171 (1) (2001) 357-372.

[16] C. J. García-Cervera, Z. Gimbutas, W. E, Accurate numerical methods for micromagnetics simulations with general geometries, Journal of Computational Physics 184 (1) (2003) 3752.

[17] F. Preisach, Über die magnetische Nachwirkung, Zeitschrift für Physik 94 (5-6) (1935) $277-302$.

[18] A. Globus, Universal hysteresis loop for soft ferrimagnetic material, Proc. Europ. Physical Society, Conference on Soft Magnetic Material 2 (1975) 233.

[19] D. C. Jiles, D. L. Atherton, Theory of ferromagnetic hysteresis, Journal of Magnetism and Magnetic Materials 61 (1986) 48-60.

[20] E. C. Stoner, E. P. Wohlfarth, A mechanism of magnetic hysteresis in heterogeneous alloys, IEEE Transactions on Magnetics 27 (4) (1991) 3475-3518.

[21] F. Liorzou, B. Phelps, D. L. Atherton, Macroscopic models of magnetization, IEEE Transactions on Magnetics 36 (2) (2000) 418-428.

[22] G. P. Carman, M. Mitrovic, Nonlinear constitutive relations for magnetostrictive materials with applications to 1-D problems, Journal of Intelligent Material Systems and Structures 6 (5) (1995) 673-683. 
[23] X. J. Zheng, L. Sun, A nonlinear constitutive model of magneto-thermo-mechanical coupling for giant magnetostrictive materials, Journal of Applied Physics 100 (6) (2006) 063906.

[24] W. D. Armstrong, An incremental theory of magneto-elastic hysteresis in pseudo-cubic ferro-magnetostrictive alloys, Journal of Magnetism and Magnetic Materials 263 (2003) $208-218$.

[25] P. G. Evans, M. J. Dapino, Efficient magnetic hysteresis model for field and stress application in magnetostrictive Galfenol, Journal of Applied Physics 107 (6) (2010) 063906.

[26] S. Chakrabarti, Modeling of 3D magnetostrictive systems with application to Galfenol and Terfenol-D transducers, Ph.D. Dissertation, The Ohio State University, 2011.

[27] S. Chakrabarti, M. J. Dapino, Nonlinear finite element model for 3D Galfenol systems, Smart Materials and Structures 20 (10) (2011) 105034.

[28] Z. Deng, M. J. Dapino, Characterization and finite element modeling of Galfenol minor flux density loops, Proc. SPIE, Behavior and Mechanics of Multifunctional Materials and Composites 8689 (2013) 86890V-11.

[29] H. Tari, J. J. Scheidler, M. J. Dapino, Robust solution procedure for the Discrete EnergyAveraged Model on the Calculation of 3D Hysteretic magnetization and magnetostriction of iron-gallium alloys, Journal of Magnetism and Magnetic Materials 384 (0) (2015) 266-275.

[30] H. Tari, S. S. Santapuri, M. J. Dapino, Efficient and robust nonlinear model for smart materials with application to composite magnetostrictive plates, Smart Materials and Structure, under review .

[31] H. Tari, H. J. Su, Complete solution to the eight-point path generation of slider-crank four-bar linkages, Journal of Mechanical Design 132 (8) (2010) 081003.

[32] H. Tari, H. J. Su, T. Y. Li, A constrained homotopy technique for excluding unwanted solutions from polynomial equations arising in kinematics problems, Mechanism and Machine Theory 45 (6) (2010) 898-910.

[33] B. Banerjee, T. F. Walsh, W. Aquino, M. Bonnet, Large scale parameter estimation problems in frequency-domain elastodynamics using an error in constitutive equation functional, Computer Methods in Applied Mechanics and Engineering 253 (2013) 60-72. 
[34] T. Ligurský, Y. Renard, A continuation problem for computing solutions of discretised evolution problems with application to plane quasi-static contact problems with friction, Computer Methods in Applied Mechanics and Engineering 280 (2014) 222-262.

[35] H. Tari, M. J. Dapino, Fast and globally convergent nonlinear system model for 3D magnetostrictive systems, ASME Conference on Smart Materials, Adaptive Structures and Intelligent Systems, Rhode Island, USA, 2014.

[36] M. J. Box, D. Davies, W. H. Swann, Nonlinear optimization techniques (Monograph No. 5: Mathematical and Statistical Techniques for Industry), Imperial Chemical Industries Limited, Oliver \& Boyd, 1969.

[37] S. Santapuri, J. J. Scheidler, M. J. Dapino, Two-dimensional dynamic model for composite laminates with embedded magnetostrictive materials, Composite Structures 132 (0) (2015) $737-745$.

\section{Appendix A. Detailed Derivation of a Selection of the Derivative Terms}

The derivatives of the Gibbs free energy (eq. (9)) have major roles in the analytical reduction of the derivation of the subsequent derivative terms, which have already been presented in the foregoing sections. Therefore, a step by step derivation procedure for such key terms is given as follows.

First, letting $\operatorname{tr}()$ stand for the trace of a matrix, we rewrite the Gibbs free energy given by eq. (9) as

$$
\stackrel{k}{G}=\frac{1}{2} \stackrel{k}{\mathbf{m}} \cdot \mathbf{K} \stackrel{k}{\mathbf{m}}-\left(K \stackrel{k}{\mathbf{c}}+\mu_{0} M_{s} \mathbf{H}\right) \cdot \stackrel{k}{\mathbf{m}}+\stackrel{k}{K_{0}}+\frac{3}{2} c_{0} \lambda_{100} \operatorname{tr}(\mathbf{T}), \quad k \in \pm\left\{1, \ldots, \frac{r}{2}\right\}
$$

which leaves the direct model unchanged, as we are shifting the base energy of all of the easy axes the same amount. Differentiating the foregoing equation with respect to field and stress gives

$$
\begin{aligned}
\frac{\partial \stackrel{k}{G}}{\partial H_{p}} & =\frac{\partial \stackrel{k}{\mathbf{m}}}{\partial H_{p}} \cdot \mathbf{K} \stackrel{k}{\mathbf{m}}-\mu_{0} M_{s} m_{p}-\left(K \stackrel{k}{\mathbf{c}}+\mu_{0} M_{s} \mathbf{H}\right) \cdot \frac{\partial \stackrel{k}{\mathbf{m}}}{\partial H_{p}} \\
& =\frac{\partial \stackrel{k}{\mathbf{m}}}{\partial H_{p}} \cdot\left(\mathbf{K} \stackrel{k}{\mathbf{m}}-K \stackrel{k}{\mathbf{c}}-\mu_{0} M_{s} \mathbf{H}\right)-\mu_{0} M_{s} m_{p}=\stackrel{k}{\gamma} \frac{\partial \stackrel{k}{\mathbf{m}}}{\partial H_{p}} \cdot \stackrel{k}{\mathbf{m}}-\mu_{0} M_{s} m_{p}=-\mu_{0} M_{s} m_{p},
\end{aligned}
$$


and

$$
\begin{aligned}
& \frac{\partial \stackrel{k}{G}}{\partial T_{i}}=\frac{\partial \stackrel{k}{\mathbf{m}}}{\partial T_{i}} \cdot \mathbf{K} \stackrel{k}{\mathbf{m}}+\frac{1}{2} \stackrel{k}{\mathbf{m}} \cdot \frac{\partial \mathbf{K}}{\partial T_{i}} \stackrel{k}{\mathbf{m}}-\frac{\partial \stackrel{k}{\mathbf{m}}}{\partial T_{i}} \cdot\left(K \stackrel{k}{\mathbf{c}}+\mu_{0} M_{s} \mathbf{H}\right)+\frac{3}{2} c_{0} \lambda_{100} \frac{\partial \operatorname{tr}(\mathbf{T})}{\partial T_{i}} \\
& =\frac{\partial \stackrel{k}{\mathbf{m}}}{\partial T_{i}} \cdot \mathbf{K} \stackrel{k}{\mathbf{m}}+\frac{1}{2} \stackrel{k}{\mathbf{m}} \cdot \frac{\partial \mathbf{K}}{\partial T_{i}} \stackrel{k}{\mathbf{m}}-\frac{\partial \stackrel{k}{\mathbf{m}}}{\partial T_{i}} \cdot(\mathbf{K}-\stackrel{k}{\gamma} \mathbf{I}) \stackrel{k}{\mathbf{m}}+\frac{3}{2} c_{0} \lambda_{100} \frac{\partial \operatorname{tr}(\mathbf{T})}{\partial T_{i}} \\
& =\frac{1}{2} \stackrel{k}{\mathbf{m}} \cdot \frac{\partial \mathbf{K}}{\partial T_{i}} \stackrel{k}{\mathbf{m}}+\frac{3}{2} c_{0} \lambda_{100} \frac{\partial t r(\mathbf{T})}{\partial T_{i}} \\
& =-\left\{\begin{array}{lr}
\frac{3}{2} \lambda_{100}\left(m_{i}^{k} m_{i}^{k}-c_{0}\right), & i \in\{1,2,3\} \\
3 \lambda_{111} \stackrel{k}{m_{1}} m_{2}, & i=4 \\
3 \lambda_{111} \stackrel{k}{m_{2}} \stackrel{k}{m}_{3}, & i=5 \\
3 \lambda_{111} \stackrel{k}{m_{3}} m_{1}, & i=6
\end{array}\right\}=-\stackrel{k}{\lambda_{i},}
\end{aligned}
$$

where we use the identities

$$
\frac{\partial \stackrel{k}{\mathbf{m}}}{\partial H_{p}} \cdot \stackrel{k}{\mathbf{m}}=0 \quad \text { and } \quad \frac{\partial \stackrel{k}{\mathbf{m}}}{\partial T_{i}} \cdot \stackrel{k}{\mathbf{m}}=0,
$$

which originate from differentiating eq. (15b) with respect to field and stress. 
Figure 4: Galfenol tip displacements with $I_{0}=0.8 \mathrm{~A}$ and $\omega=50 \mathrm{~Hz}$ for two periods: (a) and (b) x-component; (c) and (d) y-component; and (e) and (f) z-component. 
(a)

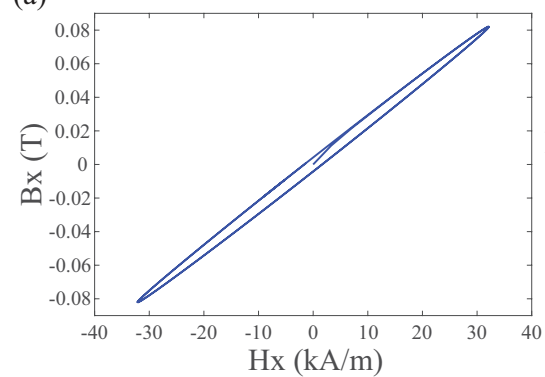

(b)

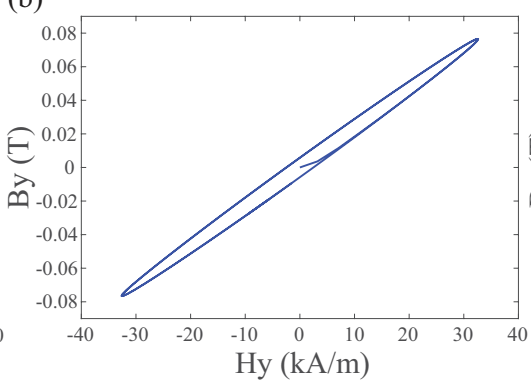

(c)

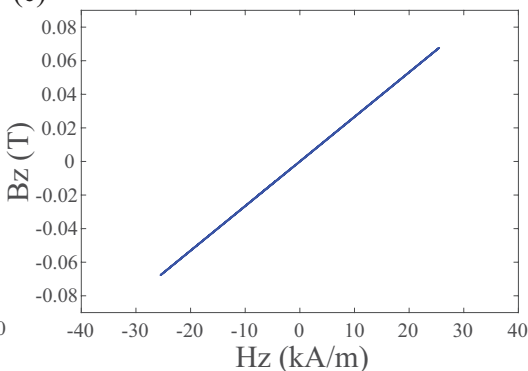

Figure 5: Magnetic flux density-magnetic field curves at the Galfenol tip with $I_{0}=0.8 \mathrm{~A}$ and $\omega=50 \mathrm{~Hz}$ for two periods: (a) x-component, (b) y-component, and (c) z-component.

(a)

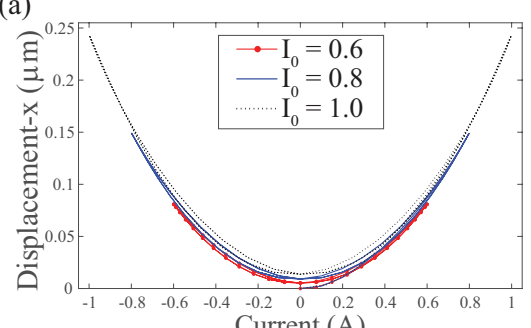

(b)

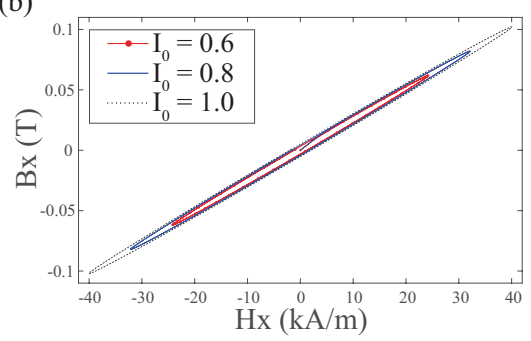

(c)

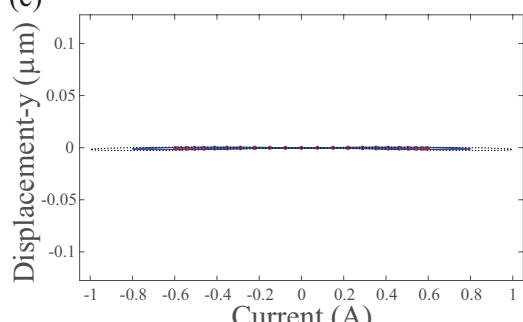

(d)

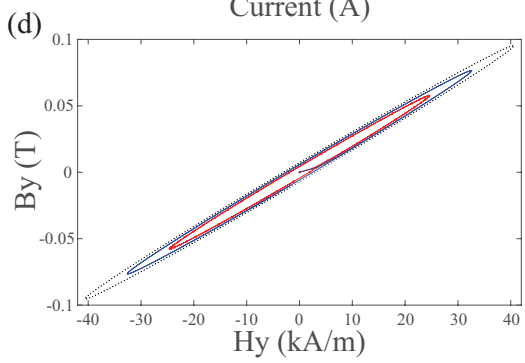

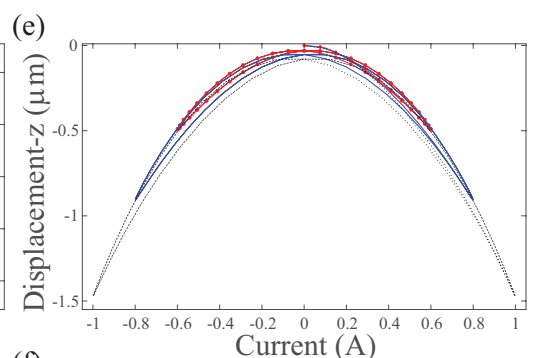

(f)

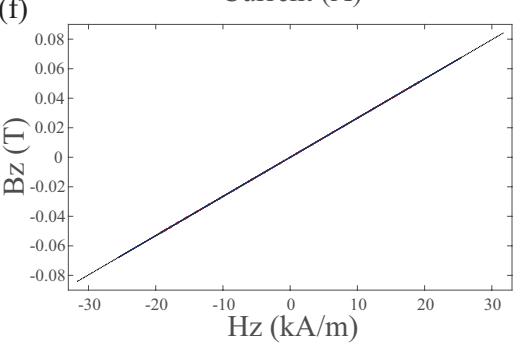

Figure 6: Displacement-current and magnetic flux density-magnetic field simulation curves at the Galfenol tip for different current magnitudes at $\omega=50 \mathrm{~Hz}$ for two periods: (a) and (b) x-component; (c) and (d) y-component; and (e) and (f) z-component. 

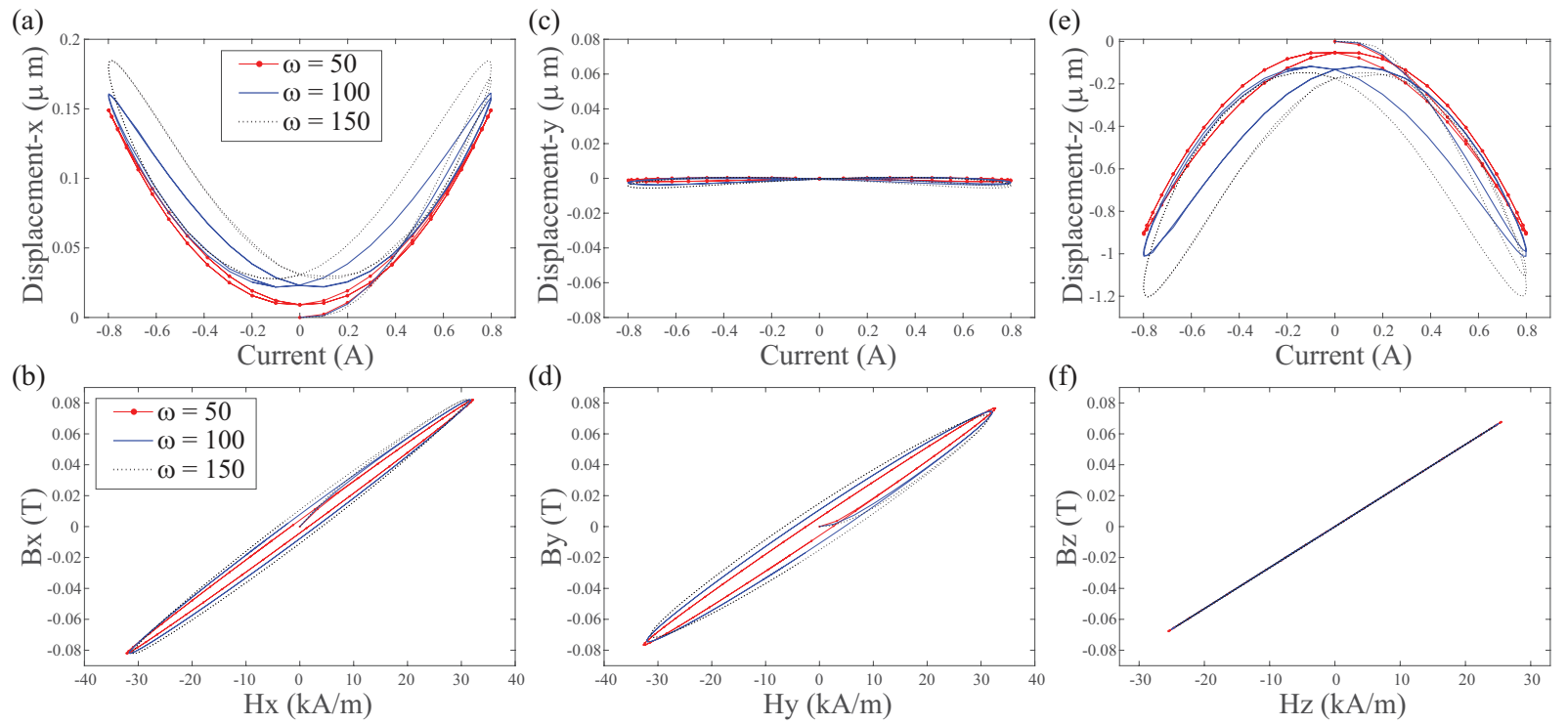

Figure 7: Displacement-current and magnetic flux density-magnetic field simulation curves at the Galfenol tip for different frequencies at $I_{0}=0.8$ A for two periods: (a) and (b) x-component; (c) and (d) y-component; and (e) and (f) z-component. 